\title{
MODELS OF DYNAMIC CONTACT OF A 2D THERMOELASTIC BAR
}

\author{
MEIR SHILlOR \\ Oakland University, Department of Mathematics and Statistics, Rochester, Michigan, USA \\ e-mail: shillor@oakland.edu
}

\begin{abstract}
This work is based on a part of the plenary lecture I gave in the PCM-CMM-2019 conference in Krakow, Poland. It presents a new mathematical model for a thermoelastic 2D bar and proposes three problems for the processes of: (i) dynamic contact of the bar with an obstacle below it; (ii) vibrations of the right end between two stops; and (iii) debonding of two bars because of vibrations, humidity and thermal effects. The models are new and questions of existence of weak solutions, analysis of the solutions, effective numerical methods and simulations, as well as possible control, are unresolved yet.
\end{abstract}

Keywords: 2D bar, dynamic contact, Barber's heat exchange condition, debonding, humidity diffusion

\section{Introduction}

This work is based in part on the plenary lecture I gave in the PCM-CMM-2019 conference held in Krakow, Poland, 8-12 September 2019.

The 2D bar was introduced in (Gao and Russell, 1994; Gao, 1998) and derived rigorously in (Sofonea and Shillor, 2018), where the existence of the weak solution to quasistatic contact was established, while numerical simulations of quasistatic contact between the bar and a reactive foundation can be found in (Barboteu et al., 2017). Starting with a 3D thermoelastic system and using symmetry and the smallness of the thickness, a cross section of a thermoelastic plate is obtained. The interest in the new bar system lies in the fact that while the horizontal displacement field $u=u(x, y, t)$ depends on $x$ and $y$, the vertical displacement depends only on the horizontal coordinate, $w=w(x, t)$, which simplifies the mathematical structure, making it a $1.5 \mathrm{D}$ system, and it makes the contact conditions more transparent.

Currently, we do not have enough computational experience to determine if the new system is substantially better than a simple 2D thermoelastic system. However, the mathematics is interesting and worth pursuing further, especially when various processes involved in contact are included.

For the considerable progress in the Mathematical Theory of Contact Mechanics, we refer to the following publications (Duvaut and Lions, 1976; Eck et al., 2005; Frémond, 2002; Han and Sofonea, 2002; Migórski et al., 2013, 2018; Shillor et al., 2004; Sofonea et al., 2006) and the host of references therein. We note here that these references are only a tip of the iceberg.

The basic dynamic thermoelastic system is described in Section 2, following (Sofonea and Shillor, 2018), with the addition of heat conduction. Section 3 describes dynamic contact of the bar with a reactive foundation, with friction, frictional heat generation and Barber's heat exchange set-inclusion condition. A model for the vibrations of the bar's end between two reactive stops is presented in Section 4. The final model describes the process of debonding of two adhesively bonded bars caused by mechanical vibrations, humidity and thermal effects. It is described in Section 5. This is an extension of the model that was introduced, analyzed and simulated in (Kuttler et al., 2017). 
Following each model we present a number of unresolved questions that are of interest for further research.

\section{The $2 \mathrm{D}$ bar dynamic equations}

We present a short description of the model equations, since the details of the derivation can be found in (Barboteu et al., 2017; Sofonea and Shillor, 2018). We consider a thermoelastic 3D rectangular solid which in an undeformed reference configuration occupies the region $\mathcal{B}=(0, L) \times(-h, h) \times(-\infty,+\infty)$ in $\mathbb{R}^{3}$, which is a plate of length $L$ and thickness $2 h$. Assuming that the system is under plane strain, we do not need the $z$ coordinate, and so the setting is $2 \mathrm{D}$ and we use $x, y$ for the spatial variables, $t$ for time, and we refer to the domain $\Omega=(0, L) \times(-h, h)$ as a $2 \mathrm{D}$ bar.

We denote by $u(x, y, t)$ the horizontal displacement of the bar, and using symmetry and a simple assumption we let $w(x, t)$ be the vertical displacement of the bar (represented by its central horizontal axis), and we let $\theta(x, y, t)$ be the temperature. Moreover, we use the indices $x$, $y, t$ to denote partial derivatives. We note that this is actually a $1.5 D$ system since $w$ depends only on $x$.

In all the models below, the bar is clamped on $\Gamma_{D}=\{0\} \times(-h, h)$, so the displacement field vanishes there and the temperature is prescribed, $\theta_{D}$; on the top, $\Gamma_{N}=(0, L) \times\{h\}$, it is subjected to distributed surface tractions of density $\mathbf{p}=[p, q]$ and the ambient temperature $\Theta_{a}$. We specify the other boundary conditions below as they relate to the different models. We denote by $\mathbf{n}$ the normal vector to $\Omega$ and by $\nu$ and $\tau$ the normal and tangential components of vectors and tensors, respectively. The setting is depicted in Fig. 1. For the sake of generality, we let $\mathbf{f}=[f(x, y, t), 0]$ be a possible body force.

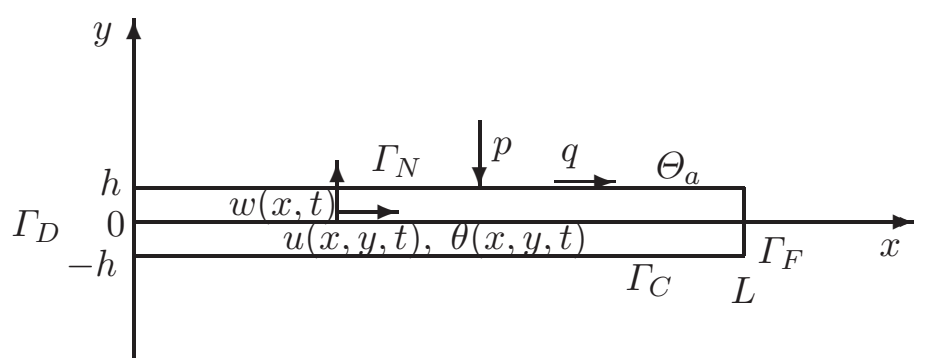

Fig. 1. The dynamic setting of the bar

Next, we describe the thermoelastic constitutive condition and present the equations of motion. The strain tensor in two-dimensions and plane strain is

$$
\boldsymbol{\varepsilon}(\mathbf{u})=\left[\begin{array}{cc}
u_{x} & \frac{1}{2}\left(u_{y}+w_{x}\right) \\
\frac{1}{2}\left(u_{y}+w_{x}\right) & 0
\end{array}\right]
$$

Therefore, $\operatorname{tr} \varepsilon(\mathbf{u})=u_{x}$; and using the usual thermoelastic constitutive law shows that the stress tensor is given by

$$
\boldsymbol{\sigma}=\left[\begin{array}{cc}
E u_{x}-\alpha \theta & G\left(u_{y}+w_{x}\right) \\
G\left(u_{y}+w_{x}\right) & (E-2 G) u_{x}-\alpha \theta
\end{array}\right]
$$

Here, $E$ is the Young modulus, $G$ is the shear modulus and $\alpha$ is the scaled coefficient of thermal expansion. We note that the strain is two-dimensional while the stress is actually three-dimensional, however, below we disregard the stress in the $z$-direction $\sigma_{z z}=(E-2 G) u_{x}-\alpha \theta$ 
since the strain and stress components do not depend on $z$. Let $\rho$ be the 2D material density, $c_{t h}$ the heat capacity, $\alpha$ the coefficient of thermal expansion and $\kappa$ the thermal conductivity, all assumed to be positive constants.

Then, we write the dynamic equation of motion as

$$
\rho \mathbf{u}_{t t}-\nabla \cdot \boldsymbol{\sigma}=\mathbf{f}
$$

the heat conduction equation

$$
\rho c_{t h} \theta_{t}-\kappa \nabla^{2} \theta+\alpha \Theta_{a} \nabla \cdot\left(u_{t}, w_{t}\right)=Q
$$

where $Q$ is a volume heat source, such as Joule heating caused by an electrical current.

Using stress tensor (2.1) and the equations above, we obtain the PDEs system and boundary and initial conditions that are common to all the models below

$$
\begin{aligned}
& \rho u_{t t}-E u_{x x}-G u_{y y}+\alpha \theta_{x}=f \\
& 2 \rho w_{t t}-G w_{x x}-(E-G) u_{x y}+\alpha \theta_{y}=0 \\
& \rho c_{t h} \theta_{t}-\kappa\left(\theta_{x x}+\theta_{y y}\right)+\alpha \Theta_{a} u_{x t}=Q
\end{aligned}
$$

where $(x, y) \in \Omega, t \in[0, T]$.

Since the bar is clamped on $\Gamma_{D}$ and has the temperature of the support $\theta_{D}$, we have

$$
u(0, y, t)=w(0, t)=0 \quad \theta(0, y, t)=\theta_{D}(y, t)
$$

for $y \in[-h, h]$, and $t \in[0, T]$.

The initial conditions are

$$
u=u_{0}(x, y) \quad w=w_{0}(x) \quad \theta=\theta_{0}(x, y)
$$

where $u_{0}$ and $\theta_{0}$ are defined on $\Omega$ and $w_{0}$ on $[0, L]$.

In what follows, we describe new models for three contact processes involving the $2 \mathrm{D}$ bar.

\section{Model of dynamic contact with an obstacle}

The mathematical model for the quasistatic process of frictional contact between the 2D bar and an obstacle below it, however without any thermal effects, was studied in (Sofonea and Shillor, 2018) where the bar was introduced and the existence of a weak or variational solution established. Then, it was simulated numerically in (Barboteu et al., 2017), where the numerical method for its computer approximations and results of some interesting simulations can be found.

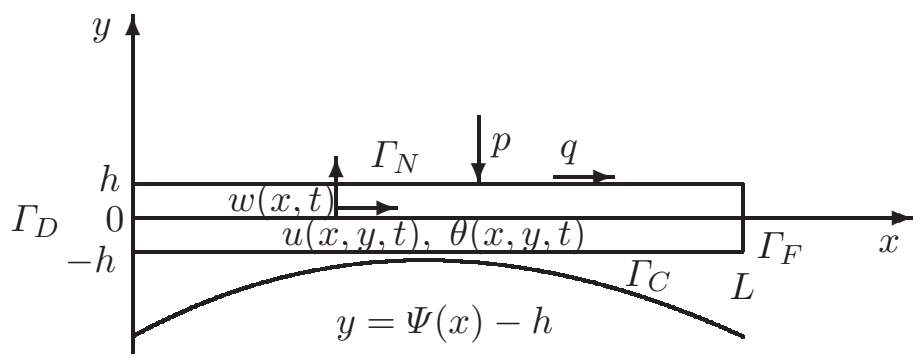

Fig. 2. The bar; $\Gamma_{C}$ is the potential contact surface and $\Psi$ describes the obstacle or foundation

Here, we present the model for the dynamic frictional contact between the bar and a reactive foundation that includes thermal effects. The setting is depicted in Fig. 2. We let the foundation 
or an obstacle be represented by $y=\Psi(x)-h$, below the bar, and then $\Gamma_{C}=(0, L) \times\{-h\}$ is the potential contact surface where the bar may contact the obstacle.

The equations for the process are given in (2.2) and the initial conditions in (2.4), while the boundary condition on $\Gamma_{D}(x=0)$ is $(2.3)$.

We turn to the other boundary conditions in this model. The outward unit normal at the boundary $\Gamma_{F}(x=L)$ is given by $\mathbf{n}=[1,0]$. Therefore, using $(2.1)$, we deduce that $\boldsymbol{\sigma} \mathbf{n}=\left[E u_{x}-\alpha \theta, G\left(u_{y}+u_{x}\right)\right]$, and thus, the boundary condition on $\Gamma_{F}$ can be written

$$
\begin{array}{ll}
u_{x}(L, y, t)=\alpha \Theta_{a} & u_{y}(L, y, t)+w_{x}(L, y, t)=0 \\
\theta(L, y, t)=\Theta_{a} &
\end{array}
$$

for $y \in[-h, h]$ and $t \in[0, T]$.

Similarly, the outward unit normal on $\Gamma_{N}(y=h)$ is $\mathbf{n}=[0,1]$. Therefore, using (2.1), we deduce that the tractions on this surface are given by $\boldsymbol{\sigma} \mathbf{n}=\left[G\left(u_{y}+w_{x}\right),(E-2 G) u_{x}-\alpha \theta\right]$. Therefore

$$
\begin{aligned}
& G\left(u_{y}(x, h, t)+w_{x}(x, t)\right)=q(x, t) \quad(E-2 G) u_{x}(x, h, t)=p(x, t)+\alpha \Theta_{a} \\
& \theta(x, h, t)=\Theta_{a}
\end{aligned}
$$

for $x \in[0, L]$, and $t \in[0, T]$. Here, $\Theta_{a}$ is the ambient temperature assumed to be constant.

We turn to the contact conditions. We recall that the normal and tangential components of the displacement field are given by $u_{n}=\mathbf{u} \cdot \mathbf{n}$ and $\mathbf{u}_{\tau}=\mathbf{u}-u_{\nu} \mathbf{n}$, respectively. Also, the normal and tangential components of the stress field are given by

$$
\sigma_{n}=(\boldsymbol{\sigma} \mathbf{n}) \cdot \mathbf{n} \quad \boldsymbol{\sigma}_{\tau}=\boldsymbol{\sigma} \mathbf{n}-\sigma_{n} \mathbf{n}
$$

Similar arguments based on (2.1) and (3.3) yield

$$
\sigma_{n}=(E-2 G) u_{x}-\alpha \theta \quad \sigma_{\tau}=\left[-G\left(u_{y}+w_{x}\right), 0\right]
$$

on $\Gamma_{C} \times(0, T)$. Moreover, since $\Psi$ is a negative function, we deduce that the gap between the bottom $y=-h$ and the obstacle is given by $g=-\Psi$.

We describe contact by the normal compliance condition, with stiffness $\lambda_{n c}$, which is usually a large constant, by

$$
\sigma_{n}=-\lambda_{n c}(\Psi-w(x, t))_{+}
$$

where $(\psi)_{+}=\max \{\psi, 0\}$, which is the positive part function. In this condition when there is no contact at $x$ at time $t, \Psi(x)-w(x, t)<0$ and the contact tractions is $\sigma_{n}(x,-h, t)=0$; while when in contact, $\Psi-w>0$ and the traction depends on the interpenetration of the surface $\Gamma_{C}$ of the bar into the surface of the obstacle. For further details, we refer to e.g., (Han and Sofonea, 2002; Martins and Oden, 1983; Migórski et al., 2013; Shillor et al., 2004; Sofonea et al., 2006) and the references therein.

Using (2.1) shows that the contact condition is

$$
(E-2 G) u_{x}(x,-h, t)-\alpha \theta(x,-h, t)=-\lambda_{n c}(\Psi(x)-w(x, t))_{+}
$$

We recall that Coulomb's law of dry friction is given as

$$
\left|\boldsymbol{\sigma}_{\tau}\right| \leqslant \mu\left|\sigma_{n}\right| \quad \boldsymbol{\sigma}_{\tau}=-\mu\left|\sigma_{n}\right| \frac{u_{t}}{\left|u_{t}\right|} \quad u_{t} \neq 0
$$

In this setting Coulomb's friction condition can be written as follows

$$
\begin{aligned}
& G\left|u_{y}(x,-h, t)+w_{x}(x, t)\right| \leqslant \mu \lambda_{n c}(\Psi(x)-w(x, t))_{+} \\
& G\left(u_{y}(x,-h, t)+w_{x}(x, t)\right)=-\mu \lambda_{n c}(\Psi(x)-w(x, t))_{+} \frac{u_{t}}{\left|u_{t}\right|} \quad u_{t} \neq 0
\end{aligned}
$$


We turn to the thermal conditions on $\Gamma_{C}$ using the Barber heat exchange condition. First, we consider frictional heat generation. We assume that the obstacle has the given temperature $\theta_{o b}(x)$, allowed to be different from the ambient temperature $\Theta_{a}$, and recall that the frictional heat generated during contact is given by

$$
J(x, t)=\beta\left|u_{t}(x,-h, t)\right|\left|\boldsymbol{\sigma}_{\tau}(x,-h, t)\right|
$$

where $\beta$ is a conversion constant, $u_{t}$ is the contact surface velocity and $\left|\boldsymbol{\sigma}_{\tau}\right|$ is the frictional resistance (actually, the friction bound). Using the normal compliance condition, we find that

$$
J(x, t)=\beta \mu \lambda_{n c}(\Psi(x)-w(x, t))_{+}\left|u_{t}(x,-h, t)\right|
$$

We note that $J=0$ when there is no relative motion $\left(u_{t}(x,-h, t)=0\right)$.

We turn to the heat exchange at the contacting part of $\Gamma_{C}$. Following (Andrews et al., 2009) (see also (Ahn et al., 2012; Migórski et al., 2018; Shillor et al., 2004), for more details), we introduce the variable $\gamma=\gamma(x, t)$ by

$$
\gamma(x, t)=(\Psi(x)-w(x, t))_{-}-\lambda_{n c}(\Psi(x)-w(x, t))_{+}
$$

which, when there is no contact satisfies $\gamma=(\Psi(x)-w(x, t))_{-}>0$ and measures the distance between the point $x$ of the bar and the obstacle; when there is contact $\gamma=-\lambda_{n c}(\Psi(x)-$ $w(x, t))_{+} \leqslant 0$ and it measures the contact stress at $x$.

We use $\gamma$ in Barber's heat exchange condition as follows. Let $H_{t h}(\gamma)$ denote the heat exchange coefficient graph, a decreasing function of the contact stress, a possible vertical jump segment $\left[h_{0}, h^{0}\right]$, and a decreasing function of the separation. A generic form of $H_{t h}(\gamma)$ is depicted in Fig. 3. Then, the Barber condition is

$$
-\kappa \theta_{y}(x,-h, t) \in H_{t h}(\gamma(x, t))\left(\theta(x,-h, t)-\theta_{o b}\right)+J(x, t)
$$

where $J(x, t)$ is given in (3.7). The interpretation of the inclusion is that there exists a function $h=h(x, t)$ that is a selection out of the graph, i.e.,

$$
h(x, t) \in H_{t h}(\gamma(x, t))
$$

for (a.a.) $x, t$, such that

$$
-\kappa \theta_{y}(x,-h, t)=h(x, t)\left(\theta(x,-h, t)-\theta_{o b}\right)+J(x, t)
$$

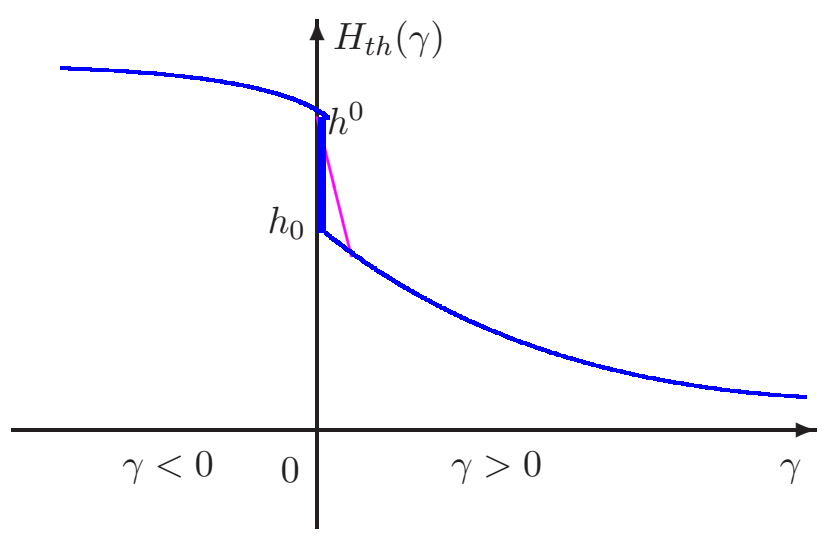

Fig. 3. The heat exchange coefficient $H_{t h}(\gamma)$; when $\gamma>0$ then it is distance, and when $\gamma<0$ it is contact stress 
We note that when contact is just established $\gamma=0$ and the heat flux is a part of the problem formulation, since then $h$ has a value in the interval $\left[h_{0}, h^{0}\right]$ that is chosen by the solution.

We note in passing that in this problem friction is controlled by the combinations $\mu \lambda_{n c} / G$.

We summarize this discussion as the following model for Dynamic frictional contact between a thermoelastic bar and a reactive foundation.

Model 1. Find the displacements $u, w$ and the temperature $\theta$ that satisfy equations (2.2), initial conditions (2.4), boundary conditions (2.3), (3.1) and (3.2) and the contact conditions on $\Gamma_{C}$

$$
\begin{aligned}
& (E-2 G) u_{x}(x,-h, t)=-\lambda_{n c}(\Psi(x)-w(x, t))_{+}+\alpha \theta(x,-h, t) \\
& G\left|u_{y}(x,-h, t)+w_{x}(x, t)\right| \leqslant \mu \lambda_{n c}(\Psi(x)-w(x, t))_{+} \\
& G\left(u_{y}(x,-h, t)+w_{x}(x, t)\right)=-\mu \lambda_{n c}(\Psi(x)-w(x, t))_{+} \frac{u_{t}}{\left|u_{t}\right|} \quad u_{t} \neq 0 \\
& -\kappa \theta_{y}(x,-h, t) \in H_{t h}(\gamma(x, t))\left(\theta(x,-h, t)-\theta_{o b}\right)+J(x, t)
\end{aligned}
$$

Here, the functions $\gamma$ and $J$ are given in (3.8) and (3.7), respectively.

The model is new and establishing the existence of its (weak or variational) solutions is the first step in its study. We remark that in view of the nonlinearities in the contact conditions, uniqueness of the solution is unlikely. Its numerical simulations may be of considerable interest, especially its noise and vibrations characteristics. Moreover, there is a need to construct efficient and convergent numerical methods for simulations of the process.

\section{Model of vibrations between two stops}

We next propose a model for frictionless vibrations of the thermoelastic bar when two stops restrict the vertical motion of its right end. Models of vibrations of the beam between two stops can be found in (Schatzman and Bercovier, 1989; Dumont et al., 2003; Andrews et al., 2009).

The equations, the initial conditions and the boundary condition on $\Gamma_{D}$ are given in Section 2 . So we turn to the rest of the conditions. For the sake of simplicity, we let the conditions on the top and bottom edges $\Gamma_{N+}$ and $\Gamma_{N-}$, see Fig. 4 , be

$$
\begin{aligned}
& G\left(u_{y}(x, \pm h, t)+w_{x}(x, t)\right)=q_{ \pm}(x, t) \quad(E-2 G) u_{x}(x, \pm h, t)=p(x, t)+\alpha \Theta_{a} \\
& \theta(x, \pm h, t)=\Theta_{a}
\end{aligned}
$$

Here, $q_{ \pm}$are the horizontal tractions, $p=p(x, t)$ is the vertical traction, and $\Theta_{a}$ is the ambient temperature.

Our interest lies in the processes involved in contact at $\Gamma_{F}$ (red vertical end in the figure), which is $x=L,-h \leqslant y \leqslant h$.

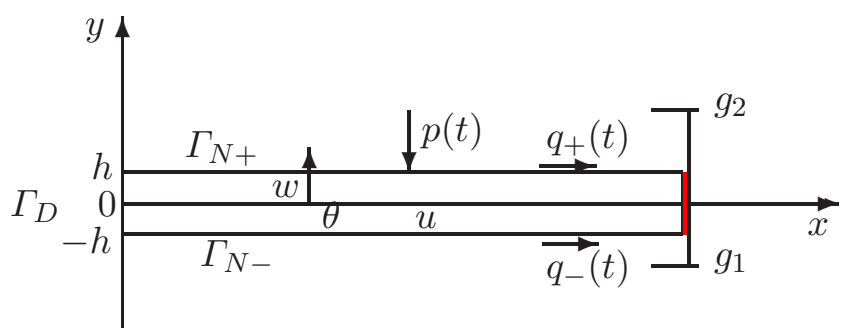

Fig. 4. The $2 \mathrm{D}$ bar; vibrations between two stops at $y=g_{1}$ and $y=g_{2}$; heat exchange takes place on the right end (red) 
Two stops are situated at $x=L$, the lower one at $y=g_{1}$ and the other one at $y=g_{2}$, where $g_{1}<0<g_{2}$. We assume that the stops are reactive and use the normal compliance condition to describe the contact at the edge. Since $\sigma_{\tau}=\sigma_{y}$, we assume that in the normal direction $(\mathbf{n}=(1,0))$ there are no tractions, hence

$$
u_{x}(L, y, t)=\alpha \Theta_{a} \quad u_{y}(L, y, t)+w_{x}(L, y, t)=0 \quad \theta(L, y, t)=\Theta_{a}
$$

for $-h<y<h$ and the temperature is the ambient temperature. In the tangential direction, we have

$$
\sigma_{y}(L, y, t)=E u_{x}(L, y, t)-\alpha \theta(L, y, t)=-\lambda_{n c}\left[\left(w(L, t)-g_{2}+h\right)_{+}-\left(g_{1}+h-w(L, t)\right)_{+}\right]
$$

This condition guarantees that when there is no contact between the edge and one of the stops, the traction vanishes, while when the bar is in contact with the top stop the reaction is downward and proportional to the interpenetration, while when in contact with the lower stop the reaction is upward. Note that $\sigma_{y} \neq 0$ only when there is contact at the top or bottom.

We summarize in the following model for Dynamic frictionless contact between a thermoelastic bar and two reactive stops at one end.

Model 2. Find the displacements $u, w$ and the temperature $\theta$ that satisfy equations (2.2), initial conditions (2.4), boundary conditions (2.3), (4.1) and (4.2) and the contact conditions on $\Gamma_{F}$

$$
E u_{x}(L, y, t)-\alpha \Theta_{a}=-\lambda_{n c}\left[\left(w(L, t)-g_{2}+h\right)_{+}-\left(g_{1}+h-w(L, t)\right)_{+}\right]
$$

The model is new and establishing the existence of its (weak or variational) solutions is the first step in its study, and to that end we need to formulate it as a variational inequality or a dynamic set-inclusion. We remark, as above, that in view of the nonlinearities in the contact conditions, uniqueness of the solution is unlikely.

Additional versions of the model that may be of interest are:

- Assume that the traction $p$ is periodic and study the noise characteristics of the contacting end and its dependence on the frequency of the tractions.

- Assume that the tractions $q_{+}$and $q_{-}$are periodic and study the noise characteristics of the contacting end and its dependence on the frequency of the tractions.

- Replace condition $(4.2)_{3}$ with the heat exchange condition

$$
-\kappa \theta_{x}(L, y, t)=h_{e x}\left(\theta(L, y, t)-\theta_{o b}\right)
$$

where $h_{e x}$ is the coefficient of heat exchange and $\theta_{o b}$ is the obstacle temperature.

- Replace the normal compliance condition with the Signorini condition

$$
\begin{aligned}
& g_{1}+h \leqslant w(L, t) \leqslant g_{2}-h \\
& w(L, t)=\left\{\begin{array}{lll}
g_{2}-h & \text { then } \quad \sigma_{y} \leqslant 0 \\
g_{1}+h & \text { then } \quad \sigma_{y} \geqslant 0
\end{array}\right. \\
& \sigma_{y}\left(\left(w(L, t)-g_{2}+h\right)\left(w(L, t)+g_{1}-h\right)\right)=0
\end{aligned}
$$

- Assume that the stops are attached to a rigid system that moves vertically as $y=\varphi(t)$, and then we replace the static stops $g_{1}, g_{2}$ with moving stops $g_{1}+\varphi(t)$ and $g_{2}+\varphi(t)$, respectively. Then, the contact condition becomes

$$
E u_{x}(L, y, t)-\alpha \Theta_{a}=-\lambda_{n c}\left[\left(w(L, t)-g_{2}+h\right)_{+}-\left(g_{1}+h-w(L, t)\right)_{+}\right]
$$

A similar problem for a Bernoulli beam was studied in (Dumont et al., 2003). 


\section{Model of debonding of two bars}

We present a new model for the debonding of two bonded bars caused by mechanical vibrations, humidity and thermal effects.

Debonding of plates with adhesive is an important process in many industrial, everyday life situations and in transportation, and too often with negative or even catastrophic consequences. A model for the debonding process of a two beam-rod system caused by vibrations, humidity and thermal effects has been constructed, analyzed and computer simulated in (Kuttler et al., 2017) where additional literature on the topic may be found. Here, we extend this model to a system of two bonded bars. Our main interest is in the dynamics of the bonding field $\beta=\beta(x, t)$ that is defined on the contact surface, $\Gamma_{B}$ in Fig. 5. It represents the fraction of active bonds and has the character of a damage variable, namely, $0 \leqslant \beta \leqslant 1$; when $\beta=1$ all the bonds of the adhesive at the point are active; when $\beta=0$ all the bonds are severed; and when $0<\beta<1$, it represents the fraction of active bonds, with the related decrease in the load carrying capacity of the adhesive.

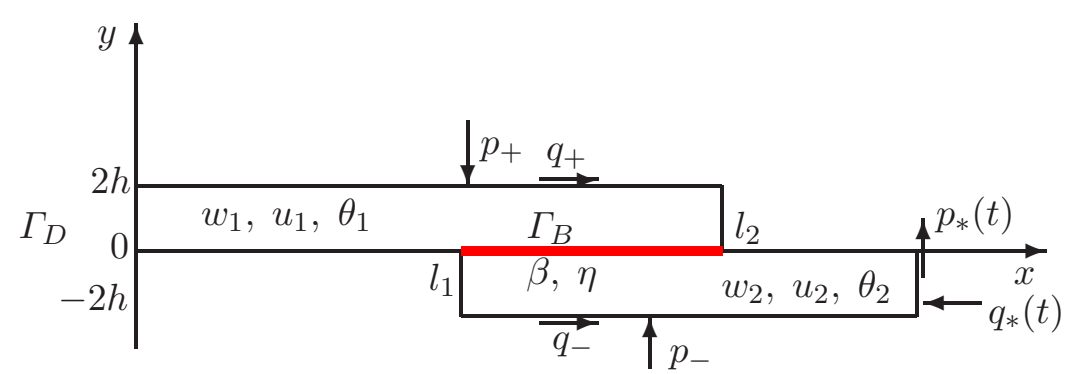

Fig. 5. Two bars in adhesive contact. The adhesive (red) occupies the interval $l_{1} \leqslant x \leqslant l_{2}, y=0$ where the functions $\beta$ - the bonding field, and $\eta$ - the humidity function are defined

The setting is as follows, Fig. 5. The top bar occupies the domain $\Omega_{1}=\left\{0<x<l_{2}, 0<y<2 h\right\}$ and the bottom bar occupies $\Omega_{2}=\left\{l_{1}<x<L,-2 h<y<0\right\}$, for $0<l_{1}<l_{2}<L$. The quantities with index $i=1,2$ refer to the bar $\Omega_{i}$, thus the horizontal displacements $u_{i}=u_{i}(x, y, t)$ are defined on $\Omega_{i}$, and so are the temperatures $\theta_{i}=\theta_{i}(x, y, t)$, and the vertical displacements $w_{i}=w_{i}(x, t)$ are defined on $\Omega_{i} \cap\{y=0\}$.

The debonding process takes place on the surface $\Gamma_{B}=\left\{l_{1}<x<l_{2}, y=0\right\}$ that is occupied by the adhesive, marked in red in Fig. 5 , where the bonding field $\beta=\beta(x, t)$ and the humidity field $\eta=\eta(x, t)$ are defined. We assume that there are no body forces acting in the system; the vertical and horizontal tractions $p_{+}=p_{+}(x, t)$ and $q_{+}=q_{+}(x, t)$ act on the top boundary of $\Omega_{1}$ while it is clamped on $\Gamma_{D}$, and free at $x=l_{2}$ and on the bottom for $0 \leqslant x<l_{1}$. The temperature on $\partial \Omega_{1}$ and $\partial \Omega_{2}$ except for $\Gamma_{B}$ is the ambient temperature $\Theta_{a}$. Similarly, the vertical and horizontal tractions $p_{-}=p_{-}(x, t)$ and $q_{-}=q_{-}(x, t)$ act on the bottom boundary of $\Omega_{2}$, it is free on $x=l_{1}$, and on the top for $l_{2} \leqslant x<L$. Tractions $p_{*}(t)$ and $q_{*}(t)$ act on the side $x=L$.

The interest in this problem lies in the debonding process on $\Gamma_{B}$, which we model as follows. The process is controlled by the debonding source function $\Phi$ that depends on the bonding field, humidity, temperature and heat fluxes from the bars and on the tractions generated by the bars. Mathematically, we write it as

$$
\Phi(x, t)=\Phi\left(\left|u_{2}-u_{1}\right|,\left(w_{1}-w_{2}\right), \beta, \eta, \theta, \theta_{y}\right)
$$

where $l_{1} \leqslant x \leqslant l_{2}$ and $y=0$. We assume that the debonding process also depends on diffusion as the field at a point is affected by what happens in neighboring points. This function contains the information about the processes and must be determined from experimental data. 
Next, we need to describe the constraint $0 \leqslant \beta \leqslant 1$, and also that $w_{2}(x, t) \leqslant w_{1}(x, t)$ on $\Gamma_{B}$. To that end, we let $I_{[0,1]}(\beta)$ and $I_{[0, \infty)}(r)$ be the indicator functions of the intervals $[0,1]$ and $[0, \infty)$, respectively, and let their subdifferential be given by

$$
\partial I_{[0,1]}(\beta)=\left\{\begin{array}{ll}
(-\infty, 0] & \text { if } \quad \beta=0 \\
0 & \text { if } 0<\beta<1 \\
{[0, \infty)} & \text { if } \beta=1
\end{array} \quad I_{[0, \infty)}(r)= \begin{cases}(-\infty, 0] & \text { if } \quad r=0 \\
0 & \text { if } \quad 0<r\end{cases}\right.
$$

The equation of evolution of the bonding field is given by

$$
\beta_{t}-k_{\beta} \beta_{x x}+\Phi \in-\partial I_{[0,1]}(\beta) \quad \beta(x, 0)=\beta_{0}(x)
$$

where $k_{\beta}$ is the bonding diffusion coefficient, and the subdifferential on the right-hand side guarantees that $0 \leqslant \beta \leqslant 1$, when $0 \leqslant \beta_{0} \leqslant 1$. Next, we assume that the humidity function evolves by nonlinear diffusion, from the edges $x=l_{1}, y=0$ and $x=l_{2}, y=0$, with the coefficient of diffusion $D$ that depends on the debonding, stress, humidity and the temperature in the region occupied by the adhesive. Thus

$$
\eta_{t}-\left[D\left(\left|u_{2}-u_{1}\right|,\left(w_{1}-w_{2}\right), \beta, \eta, \theta\right) \eta_{x}\right]_{x}=0 \quad \eta(x, 0)=\eta_{0}(x)
$$

Here, $0 \leqslant \eta_{0}(x)$ is the initial humidity in the adhesive.

The thermoelastic equations of motion in the bar $i=1,2$ are given by

$$
\begin{aligned}
& \rho_{i} u_{i t t}-E_{i} u_{i x x}-G_{i} u_{i y y}+\alpha_{i} \theta_{i x}=0 \\
& 2 \rho_{i} w_{i t t}-G_{i} w_{i x x}-\left(E_{i}-G_{i}\right) u_{i x y}+\alpha_{i} \theta_{i y}=0 \\
& \rho_{i} c_{t h i} \theta_{i t}-\kappa_{i}\left(\theta_{i x x}+\theta_{i y y}\right)+\alpha_{i} \Theta_{a} u_{i x t}=0
\end{aligned}
$$

where $(x, y) \in \Omega_{i}, t \in[0, T]$.

The balance of vertical and horizontal tractions on $\Gamma_{B}$ due to the adhesive, $\sigma_{\beta v}$ and $\sigma_{\beta h}$, is written (for the sake of simplicity) as

$$
\begin{aligned}
& \sigma_{\beta v}=K_{\beta v} \beta=k_{v}\left(w_{1}(x, t)-w_{2}(x, t)\right) \\
& \sigma_{\beta h}=K_{\beta h} \beta=k_{h}\left(\left|u_{1}(x, 0, t)-u_{2}(x, 0, t)\right|\right)
\end{aligned}
$$

Here, $k_{v}$ and $k_{h}$ are the adhesive stiffnesses. We assume that the heat flux across $\Gamma_{B}$ is proportional to the temperature difference, thus

$$
\kappa_{1} \theta_{1 y}=-\kappa_{2} \theta_{2 y}=\kappa_{\beta}\left(\theta_{1}-\theta_{2}\right)
$$

Finally, the boundary conditions for $\beta$ and $\eta$ are

$$
\beta_{x}\left(l_{1}, t\right)=\beta_{x}\left(l_{2}, t\right)=0 \quad \eta\left(l_{1}, t\right)=\eta\left(l_{2}, t\right)=\eta_{a m b}
$$

where $\eta_{a m b}$ is the ambient humidity.

Next, we let the tractions acting on the boundaries be

$$
\begin{array}{lll}
p_{+}, q_{+} & \text {on } & 0 \leqslant x \leqslant l_{2}, y=2 h \\
p_{-}, q_{-} & \text {on } & l_{1} \leqslant x \leqslant L, y=-2 h \\
p_{*}, q_{*} & \text { on } & x=L, \quad-2 h \leqslant y \leqslant 2 h
\end{array}
$$

and zero tractions everywhere else, except for $\Gamma_{D}$ where

$$
u(0, y, t)=0 \quad w(0, t)=0
$$


Finally, the initial conditions are

$$
u_{i}=u_{i 0} \quad u_{i t}=\bar{u}_{i 0} \quad w_{i}=w_{i 0} \quad w_{i t}=\bar{w}_{i 0} \quad \theta_{i}=\theta_{i 0}
$$

The model for the debonding of two adhesively jointed bars due to vibrations, humidity and thermal effects is to find the functions $\left\{u_{i}, w_{i}, \theta_{i}\right\}$ defied on $\Omega_{i}$, for $i=1,2$, and the functions $\beta$ and $\eta$, defined on $\Gamma_{B}$, such that (5.1)-(5.9) hold. Here, the initial conditions, the boundary conditions and the various coefficient functions are assumed to be known.

The problem is new, and some of its aspects will be studied theoretically and numerically in the near future.

The questions of interest, once the existence of weak solutions has been established, are:

- Construct an efficient and convergent algorithm for computer simulations.

- Find a typical debonding process.

- Study on how does the debonding depend on the excitation frequency?

- Can one get an estimate of the degree of debonding from the shift in the spectrum?

- Correlate the computer experiments with experimental data to find a possible structure for the debonding source function $\Phi$.

\section{Acknowledgement}

The author is grateful for the support of the PCM-CMM-2019 conference organizers and the grant Nonsmooth Contact Dynamics CONMECH supported by the European Union's Framework Programme for Research and Innovation Horizon 2020.

\section{References}

1. Ahn J., Kuttler K.L., Shillor M., 2012, Dynamic contact of two Gao beams, Electronic Journal of Differential Equations, 2012, 194, 1-42

2. Andrews K.T., M'Bengue M.F., Shillor M., 2009, Vibrations of a nonlinear dynamic beam between two stops, Discrete and Continuous Dynamical System, DCDS-B, 12, 1, 23-38

3. Andrews K.T., Shi P., Shillor M., Wright S., 1993, Thermoelastic contact with Barber's heat exchange condition, Applied Mathematics and Optimization, 28, 1, 11-48

4. Barboteu M., Djehaf N., Shillor M., Sofonea M., 2017, Modeling and simulations for quasistatic frictional contact of a linear 2D bar, Journal of Theoretical and Applied Mechanics, 55, $3,897-910$

5. Dumont Y., Kuttler K.L., Shillor N., 2003, Analysis and simulations of vibrations of a beam with a slider, Journal of Engineering Mathematics, 47, 1, 61-82

6. Duvaut G., Lions J.L., 1976, Inequalities in Mechanics and Physics, Springer-Verlag, Berlin

7. Eck C., Jarusek J., Krbec M., 2005, Unilateral Contact Problems: Variational Methods and Existence Theorems, CRC Press, Taylor \& Francis Group, ISBN: 9780429121210

8. Frémond M., 2002, Non-Smooth Thermomechanics, Springer

9. GAO D.Y., 1998, Bi-complementarity and duality: A framework in nonlinear equilibria with applications to the contact problems of elastoplastic beam theory, Journal of Mathematical Analysis and Applications, 221, 2, 672-697

10. Gao D.Y., Russell D.L., 1994, A finite element approach to optimal control of a 'smart' beam, [In:] International Conference on Computational Methods in Structural and Geotechnical Engineering, P.K.K. Lee, L.G. Tham and Y.K. Cheung (Eds.), Hong Kong, 135-140

11. Han W., Sofonea M., 2002, Quasistatic Contact Problems in Viscoelasticity and Viscoplasticity, Studies in Advanced Mathematics, AMS, Providence, RI and International Press, Somerville, MA 
12. Kuttler K.L., Park A., Shillor M., Zhang W., 2001, Unilateral dynamic contact of two beams, Mathematical and Computer Modelling, 34, 3-4, 365-384

13. Kuttler K.L., Shillor M., 2001, Vibrations of a beam between two stops, Dynamics of Continuous, Discrete and Impulsive Systems, 8, 1, 93-110

14. Kuttler K.L., Kruk S., Marcinek P., Shillor M., 2017, Modeling, analysis and simulations of debonding of bonded rod-beam system caused by humidity and thermal effects, Electronic Journal of Differential Equations, 2017, 301, 1-42

15. Martins J.A.C., Oden J.T., 1983, A numerical analysis of a class of problems in elastodynamics with friction, Computer Methods in Applied Mechanics and Engineering, 40, 3, 327-360

16. Migórski S., Ochal A., Shillor M., Sofonea M., 2018, Nonsmooth dynamic frictional contact of a thermoviscoelastic body, [In the special issue:] Mathematical Analysis of Unilateral and Related Contact Problems, L. Paoli and M. Shillor M. (Eds.), Applicable Analysis, 97, 8, 1228-1245

17. Migórski S., Ochal A., Sofonea M., 2013, Nonlinear Inclusions and Hemivariational Inequalities, Advances in Mechanics and Mathematics, Vol. 26, Springer, New York

18. PAOli L., Shillor M., 2018, Vibrations of a beam between two rigid stops: vector valued measures solutions, [In the special issue:] Mathematical Analysis of Unilateral and Related Contact Problems, L. Paoli and M. Shillor M. (Eds.), Applicable Analysis, 97, 8, 1299-1314

19. Schatzman M., Bercovier M., 1989, Numerical approximation of a wave equation with unilateral constraints, Mathematics of Computation, 53, 187, 55-79

20. Shillor M., 2017, Models of debonding caused by vibrations, heat and humidity, [Chapter 15 in:] Mathematical Modelling in Mechanics, Advanced Structured Materials, 69, F. dell'Isola, M. Sofonea and D. Steigmann (Eds.), Springer, Singapore, 233-250

21. Shillor M., Sofonea M., Telega J.J., 2004, Models and Analysis of Quasistatic Contact, Springer, Berlin

22. Sofonea M., Han W., Shillor M., 2006, Analysis and Approximations of Contact Problems with Adhesion or Damage, Pure and Applied Mathematics, Chapman \& Hall/CRC Press, Boca Raton, Florida

23. Sofonea M., Shillor M., 2018, Model and analysis for quasistatic frictional contact of a 2D elastic bar, Electronic Journal of Differential Equations, 2018, 107, 1-19 